\title{
Movilidad y accesibilidad universal en la arquitectura. Caso Universidad San Gregorio de Portoviejo. Ecuador
}

\author{
Andrea Nataly Bonilla Ponce \\ Danny Emir Alcívar Vélez \\ José Elvis García Muñoz \\ Alexi Jaime Carrillo Franco
}

Artículo

Invitados internacionales

Institución: Universidad San Gregorio de Portoviejo. Ecuador

\section{E-mail:}

Andrea Bonilla Ponce: andreabp4@hotmail.com Danny Alcívar Vélez: arquelenose@hotmail.com José García Muñoz: arq.elvisgarciam@gmail.com Alexi Carrillo Franco: carrilloalexi@hotmail.com

Recibido: 20 de febrero de 2018

Aceptado: 6 de diciembre de 2018

\begin{abstract}
Resumen
El trabajo de investigación desarrollado consiste en el estudio de la movilidad y accesibilidad universal en la arquitectura, aplicado en los espacios que conforman la Universidad San Gregorio de Portoviejo (USGP), institución de educación superior ubicada en la Ciudad de Portoviejo, Provincia de Manabí, en la República del Ecuador. El objetivo de este trabajo es determinar la situación actual de la movilidad y la accesibilidad universal que propician todos los espacios construidos de la USGP, mediante la aplicación de técnicas de investigación que permitirán identificar las potencialidades y debilidades que brinda el campus universitario hacia todas las personas que acceden a ella, de acuerdo a lo que establecen las leyes y normativas técnicas tanto nacionales como internacionales. La base metodológica aplicada consiste en la aplicación de fichas de observación elaboradas por la Secretaría Técnica de Discapacidades del Ecuador (SETEDIS), las cuales constituyen un instrumento útil para el diagnóstico de elementos arquitectónicos construidos y, por tanto, permiten concluir si el objeto arquitectónico posee espacios acordes a la accesibilidad desde un punto de vista integral.
\end{abstract}

Palabras clave: accesibilidad universal; arquitectura integral; inclusión; movilidad.

Mobility and universal accessibility in architecture. In University San Gregorio de Portoviejo. Ecuador

\begin{abstract}
The research consists in the study of mobility and universal accessibility in architecture applied in the spaces that make up the San Gregorio de Portoviejo University (USGP), a higher education institution located in the city of Portoviejo, Province of Manabi, in the Republic of Ecuador. According to what is established by national and international laws and technical regulations, the objective of this study is to determine the current situation of mobility and universal accessibility of all built spaces of the USGP, through the application of research techniques that will identify the potentials and weaknesses offered by the University campus to all people that access to it. The applied methodological base consists of the application of observation cards prepared by the Technical Secretary of disability of Ecuador (SETEDIS) which constitute a useful tool for the diagnostics of architectural elements built; therefore, it allows the conclusion If the architectural object has spaces according to accessibility from an integral point of view.
\end{abstract}

Keywords: universal accessibility; integral architecture; inclusion; mobility. 


\section{Introducción}

ste artículo da a conocer el estudio de la movilidad y accesibilidad universal que se dan en los espacios que conforman la Universidad San Gregorio de Portoviejo, tomando como bases fundamentales las diferentes leyes y normas nacionales e internacionales que rigen estos aspectos y que deben ser aplicados en el espacio arquitectónico para que pueda considerarse vivible e importante ante la sociedad mundial.

La contribución de este trabajo de investigación ante la problemática arquitectónica evidenciada aclara la importancia de la integral funcionalidad para todas las personas en cualesquiera de los espacios ya construidos o que en un futuro se lleguen a construir, sin el menosprecio a nadie en el que seamos considerados todos iguales.

Es fundamental que los diseños sean funcionales para todos, es decir, crear una arquitectura integral que no tenga que discriminar a nadie por sus diferencias. Estudiando el artículo de Quinn y Degener (2002) nos da a conocer que "la dignidad humana es la norma básica de los derechos humanos. Todas y cada una de las personas tienen un valor inestimable y nadie es insignificante" (. 11).

La movilidad en lo arquitectónico y urbanístico es algo muy importante ya que nos da a entender lo que comúnmente la sociedad hace en su vida diaria para desplazarse de un lugar a otro. Por otro lado, lo que respecta a la Accesibilidad Universal (AU) se enfoca en la completa integración y en la autonomía de las personas en un espacio en el que previamente se lo ha diseñado para este fin. Por tanto, es meritorio hacer conocer que este estudio se le realizará mediante la investigación de campo, utilizando fichas de observación y analizando todos los aspectos de manera conjunta en el campus de la Universidad San Gregorio de Portoviejo con el fin de conocer la situación actual de la movilidad y Accesibilidad Universal dentro de sus predios.

\section{Antecedentes}

La accesibilidad universal es una modalidad de la sociedad mundial en la que ningún individuo con diferencias entre los demás puede ser excluido del espacio físico diseñado para todos. Consultando lo que nos dice la Real Academia Española (RAE) (2014) sobre el significado de accesibilidad, tenemos que lo sugiere como "cualidad de accesible"; en cuanto a la palabra universal nos dice que "comprende o es común a todos en su especie, sin excepción de ninguno; que lo comprende todo en la especie de que se habla". Ambas palabras juntas nos dan lo que sería Accesibilidad Universal, de esta forma se enfocaría a que todo ser humano tenga el derecho de poder acceder sin ninguna barrera a cualesquiera de los espacios existentes en el conjunto urbano.

Este concepto de Accesibilidad Universal se está incrementando cada vez más a nivel mundial, pero en algunos aspectos durante la construcción son omitidos por el hecho de ser considerados por algunas personas como zonas de terreno desperdiciadas 0 un gasto mayor en la obra que incurre en el sobredimensionamiento de espacios, así como el uso de materiales especiales y de elementos de circulación con costos elevados. Estas son algunas de las características negativas que definen a estos diseños arquitectónicos como poco funcionales y de poca accesibilidad, por lo tanto, es fundamental idealizarlos, diseñarlos y construirlos para generar una mejor calidad de vida. El estudio de la situación de las personas con discapacidad debe realizarse en el espacio físico en el que se desenvuelven y en cada una de sus actividades diarias. En la Universidad San Gregorio de Portoviejo (USGP) existen personas con discapacidad de las cuales la mayoría de ellas necesitan ayuda para poder acceder a ciertas dependencias y poder realizar las actividades que comúnmente tengan que hacer.

El espacio físico en donde actualmente está la Universidad San Gregorio de Portoviejo (USGP) brinda mejores oportunidades a los ciudadanos para poder estudiar cualquiera de las carreras existentes y ser un profesional de calidad que contribuya al desarrollo de la sociedad. Este campus universitario comprende un área de $104.073,79 \mathrm{~m}^{2} \mathrm{y}$ en ella están construidos 5 edificios. En la siguiente tabla están desglosados por área de construcción de cada uno de ellos. 
Tabla 1. Área de construcción por plantas de los diferentes edificios que conforman la USGP. Fuente: Elaborada por los autores del presente artículo. Ecuador (2017).

Figura 1. Vista aérea del campus universitario de la USGP, en donde se realiza el presente estudio en el área que por ahora está construida y que comprende la universidad. Ecuador. Fuente: Imagen tomada de Google Earth y editada por los autores del presente artículo.

\begin{tabular}{|l|l|l|l|l|}
\cline { 2 - 5 } \multicolumn{1}{c|}{} & $\begin{array}{l}\text { Área de } \\
\text { construcción } \\
\text { Planta baja }\end{array}$ & $\begin{array}{l}\text { Área de } \\
\text { construcción } \\
\text { 1er Planta alta }\end{array}$ & $\begin{array}{l}\text { Área de } \\
\text { construcción } \\
\text { 2da Planta alta }\end{array}$ & $\begin{array}{l}\text { Área de } \\
\text { construcción } \\
\text { Total }\end{array}$ \\
\hline Edificio \#1 & $2.990,00$ & $1.938,44$ & $1.890,05$ & $6.818,49$ \\
\hline Edificio \#2 & $3.109,34$ & $1.911,97$ & $1.620,90$ & $6.642,21$ \\
\hline Edificio \#3 & $3.109,34$ & $1.911,97$ & $1.620,90$ & $6.642,21$ \\
\hline Edificio \#4 & $1.973,19$ & $1.293,90$ & $1.293,90$ & $4.560,99$ \\
\hline Teatro & 642,07 & - & - & 642,07 \\
\hline
\end{tabular}

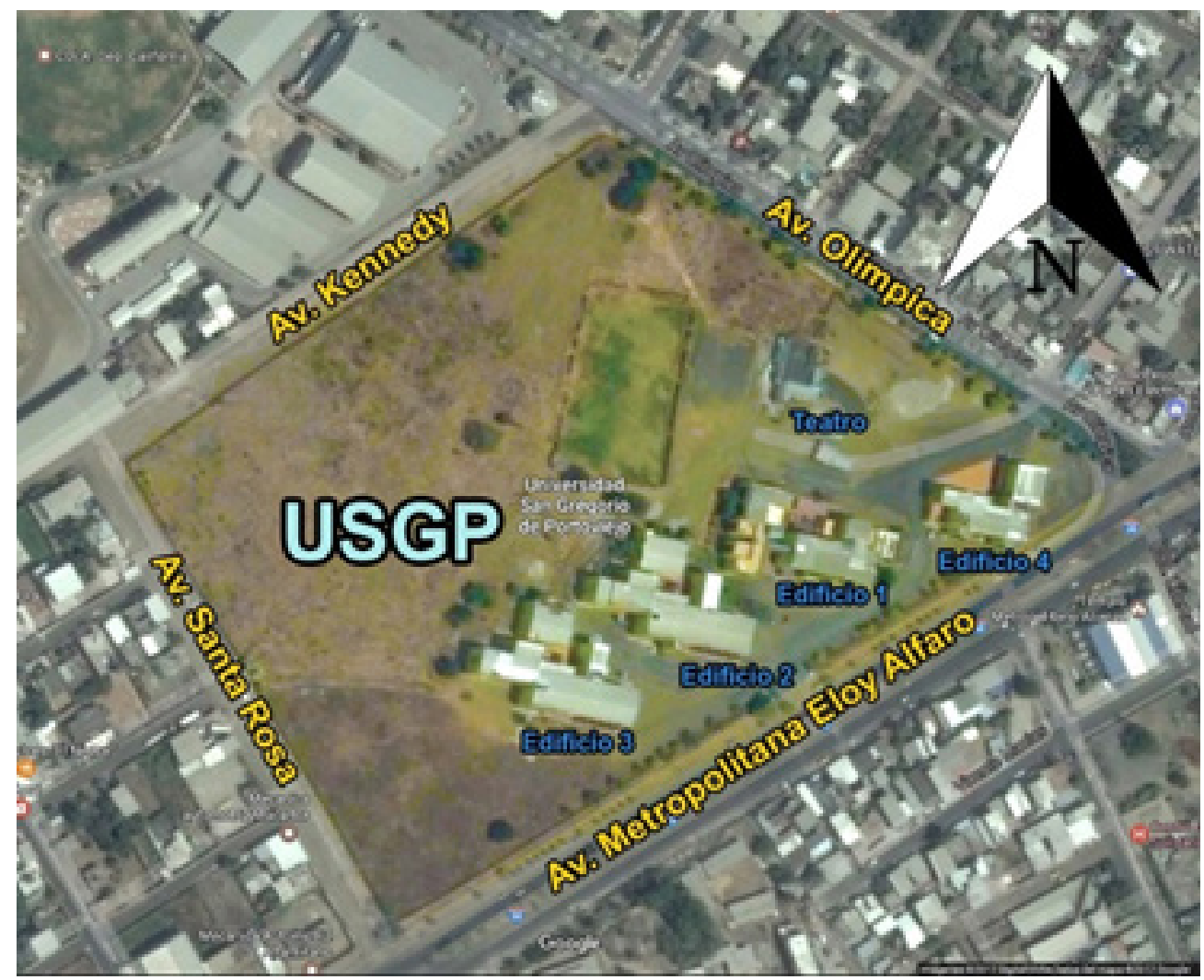

\section{Referentes teóricos}

De acuerdo a lo establecido por la Organización Mundial de la Salud, la discapacidad es un concepto en construcción que ha evolucionado paralelamente a la difusión y el ejercicio de los derechos de las personas con discapacidad. En consecuencia, en la actualidad a diferencia de épocas anteriores, la referencia a los términos sobre normalización, ambiente menos restrictivo, integración, enfoque comunitario, inclusión, ejercicio de los derechos y conceptos similares son cada vez más comunes, demostrando que el enfoque hacia las personas con discapacidad ahora se caracteriza por ser más positivo, humano y técnico.

Es así como podemos afirmar que la categoría de discapacidad es compleja, dinámica, multidimensional y, por tanto, se encuentra en constante construcción y debate; sin embargo, las personas con discapacidad y sus familias, al igual que investigadores sociales y de la salud, han identificado las barreras sociales como determinantes en la discapacidad, pues dificultan el acceso a la comunicación, información y al medio físico de este grupo de atención prioritario.

La transición que implicó pasar de una perspectiva individual y médica a una perspectiva estructural y social ha sido descrita como el viraje desde un "modelo médico" a un "modelo social", en el cual las personas con deficiencias orgánicas son consideradas personas con discapacidad por la sociedad más que por sus limitaciones funcionales.

Bajo el Modelo de Derechos Humanos e Inclusión Social, el Estado Ecuatoriano, a través de la difusión e información de instrumentos legales nacionales e internacionales, garantiza el cumplimiento de los derechos de las personas con discapacidad e incide en la colectividad al promover el reconocimiento de la discapacidad como parte de la diversidad humana y como una circunstancia de vida que implica la responsabilidad de la sociedad en general. 
Desde hace varias décadas se ha considerado el término de movilidad y accesibilidad como un elemento en constante estudio, como así lo expresa un artículo consultado en la revista en línea de Diario del Derecho (2011) que nos da a conocer que:

Movilidad, progreso y libertad se han venido entrelazando en los últimos dos siglos de la historia de las sociedades avanzadas trasformando radicalmente el escenario de aislamiento y subdesarrollo en el que transcurría la vida de la inmensa mayor parte de las personas, mediante la multiplicación de las posibilidades para desplazarse y por lo tanto de las opciones de trabajo, formación y acceso a los servicios, a la vez que las empresas generaban cada vez más riqueza y empleo. Barcos a vapor, ferrocarriles, tranvías, metropolitanos, el automóvil y la aviación comercial han ido trasformando los límites geográficos de la vida de las personas desde la estrechez de los muros de las aldeas hasta niveles de interrelación social y cultural que han ido superando una tras otra todas las fronteras. Al tiempo, y parafraseando a Larra, pegadas a las ruedas de los nuevos modos de transporte se difundía el polvo de las ideas y de la libertad, hasta extremos que ningún pensador ilustrado hubiera podido vaticinar.

Al comenzar el siglo XXI sabemos, sin embargo, que la espiral virtuosa de movilidad y desarrollo tiene límites y condicionantes muy claros. Traffic in Towns, la célebre obra de Buchanan, ya avanzó en 1963 que el coste que la calidad de vida de nuestras ciudades estaba pagando al automóvil era inasumible. Tenemos por lo tanto el reto de que cada vez más ciudadanos y ciudadanas puedan moverse fácilmente para satisfacer sus aspiraciones en relación con el empleo, la formación y los servicios, pero que ello sea compatible con tener un entorno urbano de alta calidad, en el que podamos disfrutar de un espacio urbano al servicio de las personas, con un aire limpio y con una factura energética, en especial en lo referente a los combustibles fósiles, que no nos aboque a niveles de dependencia no asumibles. (para. 1-2)

En el mismo artículo de la citada revista podemos hacer conocer que:

Nos encontramos, pues, no tan sólo ante la necesidad de solventar un problema presente, sino con la oportunidad de desarrollar un abanico de acciones preventivas en la materia, tendentes a que en las próximas décadas nuestro territorio alcance también en cuestiones de movilidad el alto nivel de excelencia al que en materia de calidad de vida y de capacidad de progreso aspira la ciudadanía. (para. 5)

El Arquitecto Rene Vallejo Aguirre, Director Metropolitano de Planificación Territorial del Distrito Metropolitano de Quito, nos comenta en una entrevista que "en la concepción de la ciudad como una organización particular de interacciones entre individuos, grupos y actividades la movilidad se constituye en elemento esencial de los sistemas urbanos, de la estructuración de las redes urbanas y de la accesibilidad y por lo tanto fundamental y necesaria para el desenvolvimiento de las actividades urbanas cotidianas" (comunicación personal, Julio 20, 2017).

En Ecuador, la historia del proceso de aceptación de las nuevas políticas de discapacidad y de los organismos que las expiden han sido fuentes de interesante reconocimiento a nivel internacional, haciendo que el país sea uno de los primeros en Latinoamérica en recibir varias distinciones por su aporte en el ámbito de la accesibilidad, la discapacidad y las políticas utilizadas. Así lo demuestra la Agenda Nacional del Consejo Nacional de Discapacidades (CONADIS) (2013), la cual en consulta en sus textos nos demuestra lo siguiente:

En el año 2001, Naciones Unidas otorga al país el premio internacional Franklin Delano Roosevelt, siendo el primer país latinoamericano en recibir esta distinción por haber realizado un trabajo destacado, de carácter intersectorial e interinstitucional que logró avances significativos, siendo referente para algunos países de América Latina y el Caribe, gracias al apoyo de RIICOTEC (Red Intergubernamental Iberoamericana de Cooperación Técnica), y del Real Patronato de España. El Consejo Nacional de Discapacidades (CONADIS) ha sido el ente rector de políticas en discapacidad y articulador de todos los sectores de la comunidad para desarrollar acciones en tres ejes temáticos: prevención, atención e integración, con el propósito de prevenir las discapacidades y elevar la calidad de vida de las personas con discapacidad, en base a la ejecución del I y II Plan Nacional de Discapacidades (2005). El 25 de septiembre de 2012, se publica la Ley Orgánica de Discapacidad en el Registro Oficial № 796 , normativa que asegura la prevención, detección oportuna, habilitación y rehabilitación de la discapacidad y garantizar la plena vigencia, difusión y ejercicio de los derechos de las personas con discapacidad. Mediante decreto del 30 de mayo del 2013, se conforma la Secretaría Técnica de Discapacidades, adscrita a la Vicepresidencia, con la finalidad de seguir adelante con la iniciativa del ex - 
vicepresidente del Ecuador Lcdo. Lenín Moreno, referente a personas con discapacidad (para. 18).

Según la CIDD (Clasificación Internacional de las Deficiencias, Discapacidades y Minusvalías) de la OMS (Organización Mundial de la Salud), la discapacidad:

Es un término general que abarca las deficiencias, las limitaciones de la actividad y las restricciones de la participación. Las deficiencias son problemas que afectan a una estructura o función corporal; las limitaciones de la actividad son dificultades para ejecutar acciones o tareas, y las restricciones de la participación son problemas para participar en situaciones vitales. (para. 1).

Por consiguiente, la discapacidad es un fenómeno complejo que refleja una interacción entre las características del organismo humano y las características de la sociedad en la que vive. (para. 2).

Analizando lo establecido en la Convención de la ONU sobre los derechos de las personas con discapacidad, (2006), podemos analizar las siguientes conceptualizaciones:

Diseño Universal:

Diseño de productos, entornos, programas y servicios que puedan utilizar todas las personas, en la mayor medida posible, sin necesidad de adaptación ni diseño especializado (art. 2).

Ajustes razonables:

Modificaciones y adaptaciones necesarias y adecuadas que no impongan una carga desproporcionada o indebida, cuando se requieran en un caso particular, para garantizar a las personas con discapacidad el goce o ejercicio, en igualdad de condiciones con las demás, de todos los derechos humanos y libertades fundamentales (art. 2).

De acuerdo a lo establecido en la guía sobre discapacidades del Consejo Nacional de Discapacidades en su capítulo 4:

Accesibilidad:

La accesibilidad es un aspecto fundamental para la inclusión social de las personas con discapacidad y un beneficio para la población en general, pues la construcción de espacios accesibles facilita y promueve la participación de la sociedad en su conjunto. Cuando se habla de accesibilidad, necesariamente se refiere a la garantía y exigibilidad de derechos, la cual se expresa a través de la eliminación de barreras arquitectónicas, urbanísticas, de transporte (accesibilidad al medio físico); así como, a la eliminación de barreras a la información y a la comunicación. La accesibilidad significa que todas las personas con y sin discapacidad pueden utilizar objetos (baños, cerraduras, pasamanos etc.), visitar un lugar o acceder a un servicio, independientemente de sus capacidades técnicas, cognitivas o físicas; la misma es indispensable e imprescindible, ya que se trata de una condición necesaria para la participación de todas las personas independientemente de las posibles limitaciones funcionales que puedan tener. (pág. 38)

Accesibilidad al medio físico:

La accesibilidad es un derecho que implica la real posibilidad de una persona de ingresar, transitar y permanecer en un lugar de manera segura, confortable y autónoma. Ello implica que las barreras de entorno físico deben ser suprimidas. Todo entorno ha de ser concebido, diseñado, construido, equipado y mantenido de forma que cada persona, independientemente de sus capacidades, pueda acceder a él y utilizarlo en condiciones de igualdad, autonomía y seguridad. La accesibilidad al entorno es esencial y su ausencia es una de las formas más sutiles de discriminación; por ello, es indispensable tomar en cuenta las Normas de accesibilidad en entornos exteriores e interiores, como otros usos específicos, priorizando la seguridad del usuario en general. (pág. 38)

En Ecuador, el Instituto Ecuatoriano de Normalización (INEN) ha creado las normas sobre accesibilidad al medio físico, las cuales constituyen una base que direcciona la planificación, ejecución, y construcción de entornos exteriores e interiores accesibles y desde estas perspectivas legales el Consejo Nacional de Discapacidades (CONADIS) y la Secretaria Técnica de Discapacidad (SETEDIS) han diseñado fichas de verificación y de evaluación de la accesibilidad a usarse en el campo, las cuales están divididas en 
las siguientes unidades de observación: aceras y circulaciones, exteriores, mobiliario urbano, rampas, orientación y señalética, seguridad y evacuación, ascensores, parada de bus, semáforos, conectividad, servicios higiénicos, estacionamientos, escaleras, espacios especializados, pasamanos, superficies, superficies acristaladas, pasillos, iluminación, pasos peatonales, tecnologías de la Información y la Comunicación (TIC's), mandos e interruptores, personal de atención, transporte, mecanismos, puertas, vados y mobiliario.

Entonces, con la aplicación de estos elementos técnicos de observación y de valoración podemos dar por sentado que el estudio de la accesibilidad a los ambientes construidos se vuelve cada vez más enérgico y que su aplicación es fundamental para que las personas con algún tipo de discapacidad tengan la confianza de que se están realizando esfuerzos para que se puedan desenvolver en el espacio físico con total normalidad. Tal y como lo dice Orellana (2011) en su tesis sobre la Accesibilidad en los edificios del campus central de la Universidad de San Carlos de Guatemala:

Unos de los mayores problemas que enfrentan las personas con discapacidad diariamente es la dificultad que presenta su movilización dentro de la sociedad, ya que la infraestructura que los rodea en su mayor parte no se encuentra en condiciones para su libre locomoción, afectando sus vidas en cuanto no pueden desenvolverse correctamente en la actual sociedad, ya que se les limita por barreras físicas, limitando varias facetas de su vida en aspectos básicos para su desarrollo como la educación.

Siendo entonces del interés de la Universidad de San Carlos de Guatemala, la realidad de su infraestructura, que de manera casi general no incluye diseños en el interior de sus edificios que proporcionen una adecuada accesibilidad. El acceso de las construcciones debería verse como la posibilidad que los mismos fueran usados en igualdad de oportunidades. Los problemas de movilización y acceso constituyen un obstáculo para el pleno ejercicio de los derechos ciudadanos, ya que no cuentan con rampas ni elevadores adecuados. (pág. vii)

\section{Metodología}

Para realizar este estudio, se implementa el siguiente plan de investigación:

Investigación de gabinete

Recolección de información bibliográfica de la movilidad y accesibilidad universal.

Análisis de datos estadísticos

Población estudiantil.

Observación de campo de los espacios construidos que conforman la USGP.

Movilidad y accesibilidad universal en los espacios construidos que conforman la USGP. Investigación de campo

Tomas de datos y observación in-situ.

Fichas de observación

Encuestas

Proceso de la investigación

Para conseguir que el estudio se realice de la mejor manera posible, se ha elaborado un consistente proceso metodológico de técnicas de investigación que lleva consigo estudios bibliográficos, cuantificación y cualificación de la movilidad y accesibilidad universal, así como la observación del espacio físico de la USGP mediante la utilización de la Guía de elaboración de planes de accesibilidad universal de la SETEDIS (2015), que nos da a conocer lo siguiente:

La «Metodología Ecuatoriana para Elaboración de Planes de Accesibilidad Universal» incluye un índice y diferentes perspectivas de análisis. Esta metodología se probó y validó en dos estudios: 1) en ciento cuarenta y nueve (149) escuelas públicas; y 2) el diagnóstico de accesibilidad en tres (3) provincias del Ecuador (Imbabura, Pastaza y Santa Elena). Esta metodología fue reconocida internacionalmente por la Design For All Foundation entre las mejores cinco prácticas del mundo, en la categoría: proyectos, propuestas, 
iniciativas, metodologías y estudios. Ecuador es el primer país latinoamericano en recibir tal distinción en la historia de los premios. (pag. 10)

Continuando con la Guía de elaboración de planes de accesibilidad universal de la SETEDIS (2015), se cita lo siguiente:

La «Metodología Ecuatoriana para elaboración de planes de Accesibilidad Universal», es una herramienta que hace posible conocer la situación en la que se encuentra determinado entorno construido, pero también muestra como proponer las alternativas para mejorar sus condiciones de Accesibilidad. Ello, permitirá a los Gobiernos Locales llevar a la práctica lo establecido en la Ley Orgánica de Discapacidades, en el marco de sus competencias.

Ponemos a su consideración esta guía, capaz de mostrar y alcanzar resultados óptimos, que dependen únicamente del carácter social y la voluntad política de quienes quieran convertir a sus territorios en mejores lugares para vivir, para todos. (pág. 10)

En esta misma guía se expresa que la metodología para determinar el Índice de Accesibilidad Universal fue el Análisis de Componentes Principales (ACP). Esta metodología consiste en la obtención de valores y vectores propios de la matriz de covarianzas muestral que se obtiene a partir de la matriz de datos (SETEDIS, 2015). Por tal razón, creemos conveniente utilizar dentro de nuestra metodología estos valores expresados en la guía para conocer, luego del estudio, el nivel de accesibilidad presente en el campus universitario; por tanto, de acuerdo con la guía tenemos que:

El Índice de Accesibilidad generado del ACP tiene:

- Un mínimo de 0 (accesibilidad del $0 \%$ en todos los grupos poblacionales y $0 \%$ en todas las condiciones de accesibilidad).

- Y un máximo de 6,56 (accesibilidad del 100\% en todos los grupos poblacionales y 100\% en todas las condiciones de accesibilidad) obtenido de la función de ACP.

Para clasificar el factor, se aplicó el concepto de intervalos de clase con dos decimales y se obtuvo:

$$
\begin{aligned}
& \text { De } 0 \text { a 2,92: BAJO } \\
& \text { De 2,92 a 4,38: MEDIO } \\
& \text { Mayor a 4,38: ALTO }
\end{aligned}
$$

Homologando los límites de cada intervalo con un porcentaje de accesibilidad, se obtuvo la siguiente escala del Índice de Accesibilidad:

Nivel Alto de Accesibilidad: Corresponde a un nivel de accesibilidad superior al 70\%.

Nivel medio de Accesibilidad: Corresponde a un nivel de accesibilidad mayor a 48\% y menor a $70 \%$.

Nivel bajo de accesibilidad: Corresponde a un nivel de accesibilidad inferior o igual al $48 \%$.

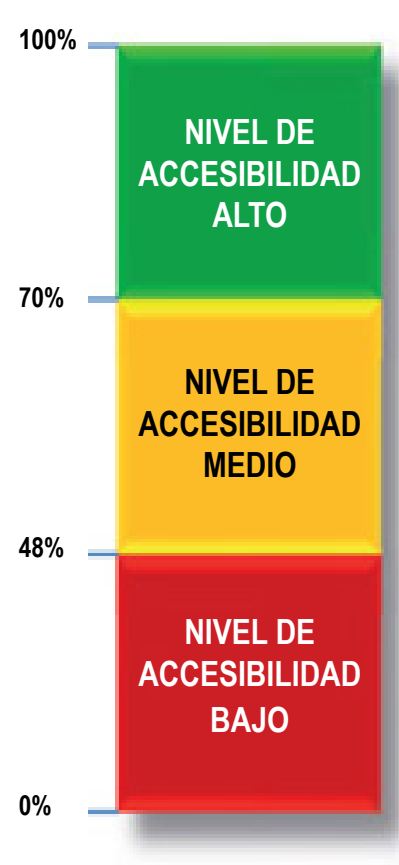

El nivel Alto corresponde a un nivel de accesibilidad superior al 70\%.

El nivel Medio corresponde a un nivel de accesibilidad mayor a $48 \%$ y menor o igual al $70 \%$.

El nivel Bajo corresponde a un nivel de accesibilidad inferior al $48 \%$. 
Tabla 2. Tabla de grupo de involucrados del presente artículo. Fuente: Elaborado por los autores del presente artículo. Ecuador. (2017).
Para que el proceso investigativo sea más efectivo, se ha calculado el tamaño de la muestra aplicando la fórmula en la que se conoce el tamaño de la población, la cual en la USGP asciende a 3007 personas (distribuidas entre estudiantes, personal administrativo y docentes) y utilizando un nivel de confianza del $95 \%$, lo que nos da como resultado un total de 171 personas como muestra para la investigación.

Este proceso metodológico lleva consigo implícito la elaboración de entrevistas y encuestas, las cuales tienen las características que sirven para ser aplicadas a todas las personas que se encuentran en los predios de la Universidad, es decir, que puede ser aplicada a las personas que tienen algún tipo de discapacidad y a aquellas que no lo tienen para así contar con información valedera y global de cada uno de los actores que viven el campus universitario.

Como ya lo mencionamos anteriormente, los organismos vinculados con la discapacidad en Ecuador han elaborado varios materiales que son instrumentos valederos para ser aplicados en el estudio de la accesibilidad en edificaciones de uso público, por tal razón hemos decidido aplicar las fichas de observación creadas por la Secretaria Técnica de Discapacidades para realizar la investigación del campo de estudio. Las fichas de observación utilizadas son las siguientes: aceras y circulaciones exteriores, ascensores, conectividad, escaleras, espacios especializados (auditorios, restaurantes, bares), iluminación mandos e interruptores, mobiliario interior, mobiliario urbano, orientación y señalética, paradas de transportes, parqueaderos, pasamanos, pasillos, pasos peatonales, para personal, puertas, rampas, seguridad y evacuación, servicios higiénicos, superficies, superficies acristaladas y Tic's accesibles.

\section{Análisis e interpretación de resultados}

Se muestran a continuación los resultados obtenidos en la investigación realizada y para esto presentaremos una tabla que muestra el grupo de personas involucradas en el presente artículo.

\begin{tabular}{|l|l|l|l|l|}
\hline 1. Grupo de involucrados & \multicolumn{1}{|c|}{} \\
\hline $\begin{array}{c}\text { Grupo / } \\
\text { individuos / } \\
\text { involucrados }\end{array}$ & $\begin{array}{c}\text { No. Población } \\
(\mathrm{n})\end{array}$ & $\begin{array}{c}\text { Tamaño de } \\
\text { muestra } \\
\text { muestreo }\end{array}$ & Método técnico \\
\hline Administradores & 116 & 1 & Intencional & Entrevista \\
\hline Administradores & 116 & Calculada (37) & Aleatoria & Encuesta \\
\hline Docentes & 175 & 3 & Intencional & Entrevista \\
\hline Docentes & 175 & Calculada (41) & Aleatoria & Encuesta \\
\hline Estudiantes & 2716 & 3 & Intencional & Entrevista \\
\hline Estudiantes & 2716 & Calculada (93) & Aleatoria & Encuesta \\
\hline
\end{tabular}

En lo referente a las encuestas, estas se realizaron en todo el campus universitario con personas que tienen como frecuencia de visita los predios universitarios aproximadamente cinco veces por semana, de las cuales se pudo obtener los siguientes resultados:

El $43 \%$ de los encuestados indicaron que en el campus universitario sí existen barreras que hacen que se evidencie el poco proceso de diseño universal arquitectónico, mientras que el 33\% desconoce de la temática en estudio.

El $51.61 \%$ de los encuestados indicaron que los edificios de la Universidad cuentan con la infraestructura necesaria para la correcta movilidad y accesibilidad universal. Todo esto debido a que son edificaciones recientemente construidas.

El $42.58 \%$ de los encuestados indicaron que los espacios en donde se realizan las actividades estudiantiles de la Universidad son adecuados.

El $44.84 \%$ de los encuestados indicaron que los espacios de servicio público como los bares-cafeterías son accesibles para las personas con discapacidad. El único 
inconveniente es que en ellos la ubicación de los mobiliarios como mesas y sillas obstaculizan la circulación.

El 56.99\% de los encuestados expresan que la biblioteca de la Universidad es accesible para las personas con discapacidad de forma independiente.

El $50.54 \%$ de los encuestados indicaron que las puertas de ingreso a las diferentes dependencias de la Universidad (secretaria general, biblioteca general y digital, policlínico, auditorios y bares - cafeterías) son accesibles de manera autónoma.

El $64.52 \%$ de los encuestados indicaron que los accesos al campus universitario brindan poca comodidad para las personas con discapacidad debido a que se encuentran solo con material pétreo compactado.

El $50.54 \%$ de los encuestados indicaron que los materiales utilizados en los pisos de las áreas exteriores de la Universidad no son apropiados para el desplazamiento de las personas con algún tipo de discapacidad.

El $66.67 \%$ de los encuestados indicaron que las paradas de buses urbanos y cantonales no están ubicadas de manera correcta, ya que se encuentran muy distantes de los predios universitarios y carecen de infraestructura adecuada.

El $51.83 \%$ de los encuestados indicaron que las señaléticas existentes en la Universidad le resultan poco útil para su movilización y para la ubicación de las diferentes actividades que se realizan en cada uno de los edificios, debido a que se encuentran en sitios pocos visibles.

En lo que concierne a la utilización de las fichas de observación aplicadas dentro del campus universitario, los predios universitarios cumplen con el $60 \%$ aproximadamente de los requisitos, por lo tanto, haremos un pequeño resumen de los datos obtenidos, debido a que son amplios y denotarían mayor cantidad de caracteres. Para ello, mostraremos unos cuantos en los que la Universidad no cumple el estándar mínimo requerido:

- La cadena de accesibilidad mantiene su continuidad, permitiendo el acceso a la edificación: la Universidad no cumple con este requisito.

- La parada de transporte público se encuentra cerca de la edificación: la Universidad no cumple con este requisito.

En caso de existir desniveles, existen vados, rebajes o rampas que facilitan la llegada de las personas con discapacidad a la entrada principal: la Universidad no cumple con este requisito.

En los bares se debe disponer de un espacio de maniobra suficiente entre las mesas (1.50m de diámetro): la Universidad no cumple con este requisito.

- Los mostradores deben disponer de un acceso lateral libre de obstáculos para personas en sillas de ruedas: la Universidad no cumple con este requisito.

- La señalización se debe colocar en lugares en los que se tomen decisiones direccionales: la Universidad no cumple con este requisito.

Los vados disponen de pavimento táctil indicador: la Universidad no cumple con este requisito.

- El ancho mínimo de las aceras es de $1.50 \mathrm{~m}$ para circulación frecuente en los dos sentidos: la Universidad no cumple con este requisito.

- Los pulsadores en los ascensores deben tener información en braille: la Universidad no cumple con este requisito.

Existen espacios de visualización para personas usuarias de sillas de ruedas y asientos accesibles en auditorios, etc.: la Universidad no cumple con este requisito.

- Las alfombras, los diferentes pavimentos de las entradas o los pavimentos táctiles indicadores pueden ayudar a las personas con discapacidad visual a localizar los mostradores de recepción: la Universidad no cumple con este requisito.

La señalización de los pasamanos es la adecuada (debe fijarse textos en relieve o textos en braille en el pasamanos: la Universidad no cumple con este requisito. 
En caso de existir desnivel, existen elementos para salvaguardar a las personas con discapacidad de estos desniveles: la Universidad no cumple con este requisito.

El personal de atención especializada tiene conocimiento como intérprete: la Universidad no cumple con este requisito.

Debe existir pavimento podotáctil de advertencia para señalizar cambios de dirección y desniveles: la Universidad no cumple con este requisito.

- Los bordes de las aceras deben estar pintados de color contrastante: la Universidad no cumple.

- La zona de información debe divisarse desde la entrada principal mediante señalética: la Universidad no cumple con este requisito.

\section{Conclusiones}

Luego de realizar el estudio de caso en los Predios del Campus Universitario, se puede concluir que en la actualidad es necesario aplicar las herramientas fundamentales del diseño universal para dotar de espacios arquitectónicos funcionales y accesibles a las instituciones y que estas sean utilizadas con autonomía por el mayor número de personas que las visitan. Tal como lo concibe Alonso (2007) es necesario crear espacios arquitectónicos con flexibilidad de uso y que además tenga un uso simple e intuitivo con información perceptible para que los usuarios puedan realizar cualquier actividad valiéndose por si mismos.

Concluyendo cada uno de los análisis realizados en las dependencias de los predios Universitarios tenemos que la Universidad San Gregorio de Portoviejo posee un porcentaje de accesibilidad del $60.33 \%$ correspondiente a un nivel de accesibilidad medio de acuerdo con lo establecido en la guía para la elaboración de planes de accesibilidad universal elaborada por la SETEDIS.

Figura 3. Índice de accesibilidad Universal. Fuente: Setedis 2015.

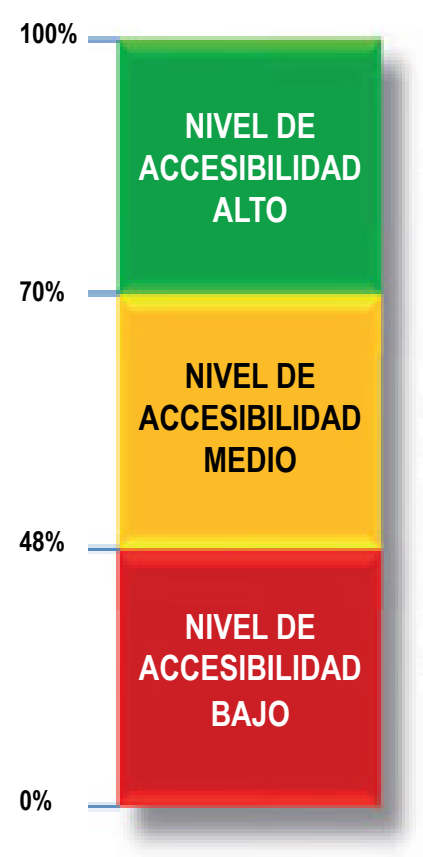

El nivel Alto corresponde a un nivel de accesibilidad superior al $70 \%$.

El nivel Medio corresponde a un nivel de accesibilidad mayor a $48 \%$ y menor o igual al $70 \%$.

El nivel Bajo corresponde a un nivel de accesibilidad inferior al $48 \%$.

A continuación, se detallan cada una de las conclusiones particulares de acuerdo al plan de investigación aplicado:

De acuerdo a los resultados obtenidos en las encuestas:

- Los accesos al campus universitario de la USGP brindan poca comodidad para las personas con discapacidad de forma independiente, además de ciertas indicaciones importantes sobre la falta de caminerías que sean apropiadas para la correcta movilidad y accesibilidad universal.

- Las señaléticas existentes en la USGP resultan poco útiles para la movilización y ubicación de las diferentes actividades que se realizan en cada uno de los edificios. Además, hacen falta señaléticas llamativas que sean visibles y que posean también 
Los edificios de la USGP cuentan con la infraestructura necesaria para la movilidad y accesibilidad universal. No obstante, no es bien aprovechado, ya que no presentan una debida conexión con el área exterior, además de no presentar un espacio de parqueo apropiado para una persona con discapacidad.

Los materiales utilizados en los pisos construidos tanto interiores como exteriores son los apropiadas para el desplazamiento de las personas con discapacidad. Sin embargo, existe la falta del sistema podotáctil y el cambio del material de la escalera con uno que evite resbalones y caídas.

- La biblioteca de la USGP no es accesible para una persona con discapacidad de forma independiente desde los demás edificios debido a la inexistencia de conexón entre los mismos. No obstante, si la persona se encuentra en el mismo edificio sí lo es, ya que cuenta con un ascensor y un suficiente espacio para la movilización.

Es necesario un ascensor adicional en el edificio \#4 para poder acceder a las demás dependencias administrativas de manera que una persona con discapacidad pueda acceder de manera autónoma.

- Las paradas de buses urbanos y cantonales existentes en la entrada de la USGP no están ubicadas de manera correcta, debido a que crean un caos vehicular con los demás carros y taxis. Sin embargo, las paradas de taxis están ubicadas de manera correcta, debido a que están a la cercanía de los estudiantes y que no crearían caos con las paradas de los buses.

- Los espacios de servicios públicos como el bar/cafetería tanto del edificio \#1 como él \#2 son accesibles para las personas con discapacidad de forma independiente. No obstante, las puertas de vidrio abatibles en los ingresos a ellos y a la Secretaría General, la Biblioteca General y Digital, el Policlínico y a los auditorios 1, 2 y 3 no son funcionales para una persona que ocupe sillas de ruedas por la fuerza que ejercen al abrirlas.

De acuerdo a los resultados obtenidos en las fichas de observación:

- No existen sistemas de comunicaciones como el braille y relieve para las personas con discapacidad visual que estén dispuestas en todo el campus universitario.

En los auditorios no existe un espacio destinado para las personas con discapacidad.

- Las mesas para los estudiantes y los mostradores de las secretarías de la USGP no cumplen con todas las dimensiones dispuestas por las fichas de observación de la SETEDIS para las personas que se desplacen en sillas de ruedas.

- Las rampas que existen en la USGP no son suficientes, además no presentan pasamanos, señal podotáctil tanto de guía como de prevención. Existen desniveles en todos los edificios de la USGP, pero estos no presentan la debida solución para que una persona con discapacidad pueda movilizarse.

Los servicios higiénicos no presentan los accesorios necesarios ni los dispositivos de emergencias y llamadas de asistencia técnica, que son considerados obligatorios por la SETEDIS.

\section{Recomendaciones}

De acuerdo a los resultados obtenidos en las encuestas:

- Es necesario remover el material de piedrillas existente en la superficie de los accesos y del área construida en el campus universitario de la USGP. Además, se necesita construir un acceso peatonal y delimitar las áreas que sean correspondientes a la movilidad de vehículos y transeúntes, al mismo tiempo que posean el sistema podotáctil guía y de prevención para que las personas con discapacidad puedan acceder de forma independiente.

- Es importante implementar señalética con colores contrastantes en la parte superior de los edificios con los respectivos números en los que fueron designados, de tal forma que sean visibles por los transeúntes y por las personas con baja visión. 
Además, los accesos a la USGP deberán contar con mapas guías con lenguaje visual, braille y táctil. Al mismo tiempo, los edificios deberán poseer planos hápticos en sus respectivos ingresos y áreas exteriores con los mismos lenguajes mencionados para que las personas con discapacidad puedan ubicarse e involucrarse más con la sociedad.

Es aconsejable que la superficie del campus universitario se le construya con un solo nivel, delimitando las áreas de aceras, caminerías y parqueaderos con bolardos. Al mismo tiempo, los estacionamientos para las personas con discapacidad podrán ser fluidas y previamente conectadas con las aceras y caminerías que deberán estar diseñadas con colores que queden contrastados con el pavimento. Además, esta tendrá implementada la señal podotáctil de guía y prevención en el que los transeúntes con discapacidad puedan movilizarse sin complicaciones de edificio en edificio en todo el campus universitario.

Es ideal que los accesos a las diferentes puertas, escaleras, ascensores, rampas y desniveles en cada uno de los edificios de la USGP posean la señal podotáctil de prevención. Al mismo tiempo estará conectada con la señal podotáctil guía que conectará con las diferentes áreas tanto interiores como exteriores del campus universitario. Además, en cada uno de los bordillos o franjas de los peldaños tendrá un material antideslizante que evite resbalones y caídas.

Es aconsejable diseñar un circuito de caminerías a un solo nivel entre los edificios 1, 2, 3 y 4 de la USGP, en el que estos no estén interrumpidos por el parqueadero para que las personas con discapacidad puedan acceder a la biblioteca de forma independiente y sin dificultades.

Es ideal que el ascensor existente en el edificio 4 tenga una conexión directa con el área administrativa en las plantas altas 1 y 2 , para que las personas con discapacidad puedan llegar a estos espacios importantes de la USGP.

Es necesario cambiar la ubicación de la parada de buses urbanos y cantonales existente en la entrada de la USGP con un diseño que sea accesible para todas las personas. Al mismo tiempo, es favorable mejorar la parada de taxis con un espacio de desaceleración en el que no entorpezca la movilización de las personas.

Es conveniente que todas las rampas posean pasamanos con lenguaje braille y de relieve. Asimismo, estarán debidamente conectadas con las caminerías y los bares/ cafeterías. Además, la puerta es ideal que sea automática, para que las personas con discapacidad puedan acceder sin mayores esfuerzos. También sería aconsejable que las puertas a la Secretaría General, la Biblioteca General y Digital, el Policlínico y los auditorios 1, 2 y 3 tengan el mismo sistema automático.

De acuerdo a los resultados obtenidos en las entrevistas:

Se deberán señalizar los accesos y vías que constan y se diseñen en la USGP, donde se delimitarán los parqueaderos para todas las personas y sus diferentes funciones. Además, se crearán dos accesos peatonales cercanos a los dos accesos vehiculares para que exista una mejor movilización tanto peatonal como vehicular.

De acuerdo a los resultados obtenidos en las fichas de observación:

Sustituir los antepechos de la USGP con pasamanos al igual que con las escaleras. Deberán poseer una superficie lisa que indiquen los números de pisos con los lenguajes visuales braille y de relieve.

Los Auditorios del edificio \#2 y \#3 deberán tener por lo menos cuatro asientos reservados para las personas con discapacidad y estarán junto a los demás. Para las personas que usen sillas de ruedas se tendrá dos espacios con dimensiones mínimas de 0,90 m x 1,40 m y estarán cercanas a las otras sillas para que puedan permanecer con su acompañante. Además, existirán cuatro sillas desplegables 0 desmontables, mientras que el edificio \#1 contará solo con los cuatro asientos reservados y todos estarán debidamente señalizados y enumerados.

Las mesas para los estudiantes deberán tener las siguientes dimensiones: la altura máxima será de 0,85 $\mathrm{m}$, la profundidad mínima será de 0,60 $\mathrm{m}$, y el ancho mínimo será de $0,90 \mathrm{~m}$. Mientras que el mostrador deberá tener una altura comprendida entre $0,80 \mathrm{~m}$ a 0,85 $\mathrm{m}$ y el espacio libre para las rodillas será como mínimo 0,70 m. 
Se tendrá que crear más rampas cercanas a los accesos y ascensores de cada edificio. Estos tendrán sus correspondientes pasamanos al igual que sus escalones aledaños con la señal podotáctil guía y de prevención.

Se deberá implementar secadores de manos y dispositivos de emergencia y llamadas de asistencia técnica con una altura comprendida entre 0,80 a 1,10 m y perchas para colgar toallas a una altura comprendida entre $1,05 \mathrm{~m}$ y $1,40 \mathrm{~m}$.

\section{Referencias Bibliográficas}

Alonso, F. (2007). Algo más que suprimir barreras: conceptos y argumentos para una accesibilidad universal. TRANS, 11, 15-30.

Consejo Nacional para la Igualdad de Discapacidades. (2013). Antecedentes Históricos. Recuperado de: http://www.planificacion.gob.ec/wp-content/uploads/ downloads/2014/09/Agenda-Nacional-para-Discapacidades.pdf.

Diario del Derecho. (2011). Ley 6/2011 de 1 de abril, de la Generalitat, de Movilidad de la Comunidad Valenciana. Disponible en: http://www.iustel.com/diario_del_derecho/ noticia.asp?ref_iustel=1048834

Ley Orgánica de Discapacidades (2012). Principios y disposiciones fundamentales. Disponible en: https://www.consejodiscapacidades.gob.ec/wp-content/uploads/ downloads/2014/02/ley_organica_discapacidades.pdf

LeyOrgánica de Discapacidades(2012).Delas personas condiscapacidad, sus derechos, garantías y beneficios. Disponible en: https://www.consejodiscapacidades.gob. ec/wp-content/uploads/downloads/2014/02/ley_organica_discapacidades.pdf

Orellana, L. (2011). Accesibilidad en los edificios del campus central de la Universidad de San Carlos de Guatemala (Tesis de tercer nivel).

Organización Mundial de la Salud (s.f.). Discapacidad. Recuperado de: http://www.who. int/topics/disabilities/es/

Quinn, G. y Degener, T. (2002). Derechos humanos y discapacidad. Uso actual y posibilidades futuras de los instrumentos de derechos humanos de las Naciones Unidas en el contexto de la discapacidad. Naciones Unidas, Nueva York y Ginebra.

Real Academia Española. (2014). Diccionario de la lengua española (23 ed.). España: Planeta Publishing.

Secretaría Técnica para la gestión inclusiva de Discapacidades (2015). Guía de elaboración de planes de accesibilidad universal. Ecuador: Vicepresidencia de la República del Ecuador. 